\title{
miR-296-5p Inhibits the Secretion of Pulmonary Surfactants in Pulmonary Epithelial Cells via the Downregulation of Wnt7b/ $\beta$ - Catenin Signaling
}

\author{
Ying-Hui Zhang, ${ }^{1}$ Ai-Ling Chen, ${ }^{2}$ Ren-Qiang Yu, ${ }^{1}$ Bei-Bei Jia, ${ }^{1}$ Dan-Ni Ye, ${ }^{1}$ Min Wang, \\ Ying-Zi Mei, ${ }^{1}$ Guang-Dong Fang, ${ }^{1}$ Shan-Yu Jiang, ${ }^{1}$ Qin Zhou, ${ }^{1}$ and Bing Zhang $\oplus^{3}$ \\ ${ }^{1}$ Department of Neonatology, The Affiliated Wuxi Maternity and Child Health Care Hospital of Nanjing Medical University, \\ Wuxi, China \\ ${ }^{2}$ Translational Medicine Laboratory, Research Institute for Reproductive Health and Genetic Diseases, The Affiliated Wuxi Maternity \\ and Child Health Care Hospital of Nanjing Medical University, Wuxi, China \\ ${ }^{3}$ Department of Gynaecology and Obstetrics, The Affiliated Wuxi Maternity and Child Health Care Hospital of Nanjing \\ Medical University, Wuxi, China
}

Correspondence should be addressed to Bing Zhang; yanghezhangbing@163.com

Received 20 July 2020; Revised 12 December 2020; Accepted 24 December 2020; Published 5 January 2021

Academic Editor: Christos K. Kontos

Copyright (c) 2021 Ying-Hui Zhang et al. This is an open access article distributed under the Creative Commons Attribution License, which permits unrestricted use, distribution, and reproduction in any medium, provided the original work is properly cited.

\begin{abstract}
Neonatal respiratory distress syndrome (NRDS) is a common disease that occurs in premature infants. However, the mechanisms underlying the disease remain unclear. microRNAs (miRNAs) have been indicated to play a crucial role in the development of NRDS. In this study, we aimed to explore the regulatory mechanisms of miR-296-5p in NRDS. The expression levels of miR296-5p in preterm infants with NRDS were determined using quantitative reverse-transcription polymerase chain reaction (RTqPCR). A549 cells were transfected with lentiviral vectors encoding miR-296-5p, and the transfection efficiency was determined using RT-qPCR. Flow cytometry and CCK8 assay were performed to measure apoptosis and proliferation of A549 cells, respectively. The protein levels of pulmonary surfactant SP-A (SFTPA1), SP-B, Wnt7b, and $\beta$-catenin were measured using western blotting. We demonstrated an upregulation of miR-296-5p in NRDS. The miR-296-5p was successfully overexpressed in A549 cells via lentivirus transfection, and the upregulation of miR-296-5p inhibited cell proliferation and secretion of SP-A and $\mathrm{SP}-\mathrm{B}$ and also induced downregulation of the Wnt7b/ $\beta$-catenin in vitro. Therefore, miR-296-5p inhibits cell proliferation and secretion of pulmonary surfactants in A549 cells via downregulation of $\mathrm{Wnt} 7 \mathrm{~b} / \beta$-catenin signaling.
\end{abstract}

\section{Introduction}

Neonatal respiratory distress syndrome (NRDS) is a serious disease that occurs in approximately $45 \%$ of mildly to moderately premature infants, which is associated with high morbidity and mortality. The major cause of this disease is lack of alveolar surfactant leading to a collapse in alveolar ventilation, which in turn gives rise to progressive dyspnea and even respiratory failure within $4-12 \mathrm{~h}$ after birth [1]. Steroid prophylactic treatment and exogenous pulmonary surfactant (PS) replacement therapy are used in treating NRDS; how- ever, the clinical efficacy of these strategies is still not satisfactory. Clinical medicine, epidemiology, and biochemistry have demonstrated that NRDS is associated with various internal and external factors such as lung dysplasia, severe infection, hypoxia, and direct damage to type II alveolar epithelial cells (AECII). Clinically, the amniotic fluid foam test, lecithin/sphingomyelin (L/S), phosphatidylglycerol determination, lamellar body count (LB), alveolar surfactant/albumin detection, and lung maturity predict the occurrence of NRDS. However, these invasive techniques are complicated, and the false positive and negative error rates associated with 
these techniques are high, thereby affecting the clinical efficacy. Therefore, the technique to identify the risk for NRDS must be improved to predict the onset of NRDS in infants.

microRNAs (miRNAs), small noncoding RNAs of $\sim 22$ nucleotides long, are known to be involved in the regulation of various cellular processes [2], such as cell differentiation and development, and may also be associated with numerous diseases [3], acting as cancer oncogenes [4]. Many studies have demonstrated that $>100$ miRNAs change in expression levels during lung development [5]. However, the mechanism for miRNA regulation of lung development remains unclear. Recent research has shown that miRNAs are differentially expressed in embryonic and adult lung tissues [68]. A study demonstrated that the inactivation of Dicer, a key component of miRNA processing, inhibits alveolar epithelial cell differentiation [9]. miRNA-17-92 overexpression has been demonstrated to promote alveolar epithelial cell proliferation and inhibit its differentiation [10]. Another study showed that miRNA-155 increased lung remodeling in knockout mice [11]. miRNA-127 overexpression leads to decreased fetal apical bud number and increased terminal and internal bud size, affecting fetal lung development [7]. These results suggest that miRNAs are closely related to lung development and may be involved in the development of NRDS. However, the role of miRNAs in NRDS has not been extensively studied. In our previous study [12], we found that the expression of miR-296 was different in fetal mice at 16 days, 19days, and 23days. However, whether miR-296 is involved in lung development remains unclear. The current research on miR-296 mainly focuses on tumors, including the proliferation of tumor cells [13-18], differentiation [14], angiogenesis [19-21], invasion [14, 22, 23], and drug resistance [24-26]. There are no reports on the role of miR-296 in NRDS. However, the literature demonstrates that miR296 is also involved in the biological processes of embryonic stem cells. It has been found that miR-296 can inhibit the nuclear stem cell gene, Nanog [27], and is significantly upregulated in epithelial cells derived from human embryonic stem cells [28]. The study found that AECII are the main stem cells present in the lungs [29]. In the mechanism of normal cell renewal and injury repair, AECII can replenish and proliferate via mitosis and can also be transdifferentiated into type I alveolar epithelial cells. However, there are no reports on their function and mechanism in the development of lung tissue. Therefore, we hypothesized that miR-296 may participate in regulating the development of AECII and contribute to the NRDS process.

In our previous study [12], three major target gene software: TargetScan6.2, PicTar, and miRanda, were used for performing functional and pathway enrichment analyses of miR-296. Downstream target genes and signaling pathways suggest that the Wnt signaling pathway is a major signaling pathway enriched with miR-296 target genes. Therefore, miR-296 may be involved in the regulation of the Wnt signaling pathway. The Wnt signaling pathway is widely distributed in invertebrates and vertebrates and is a highly evolutionarily conserved signaling pathway. Wnt signaling plays a crucial role in physiological processes, such as the early development of animal embryos [30], organ formation
[31], and tissue regeneration [32]. Binding of the Wnt molecule to a transmembrane receptor triggers the accumulation of $\beta$-catenin in the cytoplasm and its subsequent translocation into the nucleus via interaction of the receptor with Dishevelled (DVL) and a series of cytoplasmic proteins [33]. Further, nuclear transcription factors activate the transcription of downstream target genes, triggering biological effects [34]. Further studies have shown that the Wnt signaling pathway is not only crucial in embryonic development but is closely associated with the self-renewal and differentiation of various tissue stem cells. The Wnt signaling pathway is also implicated in the occurrence and development of various human diseases $[35,36]$. During lung development, blocking the Wnt signaling pathway via gene knockout of $\beta$-catenin blocks the formation of distal branches of the lungs and the differentiation of terminal airway epithelial cells, resulting in NRDS [37]. These results indicate that the Wnt signaling pathway is involved in the pathogenesis of NRDS. Therefore, we hypothesized that miRNA-296 may play an important role in NRDS by interacting with Wnt and modulating lung development, which in turn affects the formation of PS. The synthesis and secretion of PS play a crucial role in NRDS. This study may provide novel theoretical clues for the clarification and prevention of NRDS pathogenesis.

\section{Materials and Methods}

2.1. Patients and Sample Collection. The study protocol was approved by the Human Research Ethics Committee of the Affiliated Wuxi Maternity and Child Health Care Hospital of Nanjing Medical University. Written informed consent was obtained from all pregnant women involved in the study. Venous cord blood $(2 \mathrm{~mL})$ was collected during the birth of 23 preterm infants (13 without NRDS, 10 with NRDS). Serum samples were obtained from the clotted cord blood specimens and stored in a $-80^{\circ} \mathrm{C}$ ultralow freezer until further use.

2.2. Cell Culture. Cells of the A549 human lung adenocarcinoma cell line were purchased from Shanghai GeneChem Co., Ltd. (Shanghai, China) and cultured in DMEM containing $10 \%$ fetal bovine serum and $1 \%$ penicillin-streptomycin solution (Gibco, Thermo Fisher Scientific, Waltham, MA, USA). The cells were maintained in an incubator in an atmosphere containing $5 \% \mathrm{CO}_{2}$ at $37^{\circ} \mathrm{C}$. All the experiments in this study were repeated at least three times.

2.3. Construction of Plasmids. A lentiviral GV369 vector was purchased from Shanghai GeneChem Co., Ltd. The vector sequence was Ubi-MCS-SV40-EGFP-IRES-puromycin. The primer sequences of miR-296-5p were as follows: forward, $5^{\prime}$ -GAGGATCCCCGGGTACCGGGGACAGGGCTGGGAG GATTGAG-3'; and reverse, 5'-CACACATTCCACAGGC TAGGCCGCCCCAGGGGACTCAGCAG-3'. The primers contained complementary base pairs, restriction sites, and part of the $5^{\prime}$-end sequence of the target gene for PCR. Subsequently, according to the manufacturer's protocol, the designed vector was digested by AgeI and NheI restriction enzymes at $37^{\circ} \mathrm{C}$ for $30 \mathrm{~min}$ simultaneously and ligated by DNA ligase at 
$16^{\circ} \mathrm{C}$ for $2 \mathrm{~h}$. The recombinant plasmid was validated by double restriction enzyme digestion, separated by $1 \%$ PAGE, and sequenced by Sanger sequencing by Sangon Biotech Co., Ltd. The correct recombinant plasmid was termed named pGV369-296-5p.

2.4. Lentivirus Packaging. To generate lentivirus containing the recombinant plasmids, HEK 293 T cells were cultured in a cell culture plate until they reached $70-80 \%$ confluence. The media were then replaced with serum-free medium and incubated for $2 \mathrm{~h}$. Further, the cells were transfected with $20 \mu \mathrm{g}$ of the recombinant plasmid, pGV369-296-5p, along with helper plasmids, pHelper 1.0 vector plasmid $(15 \mu \mathrm{g})$ and pHelper 2.0 vector plasmid $(10 \mu \mathrm{g})$. The cells were washed with phosphate-buffered saline (PBS) $6 \mathrm{~h}$ posttransfection, and the media were replaced with DMEM containing $10 \%$ FBS. The media of HEK 293 T cells were collected $48 \mathrm{~h}$ after transfection, and the virus (L-GV369-296-5p) was harvested. The supernatant was filtered through a $0.45 \mu \mathrm{m}$ filter and centrifuged at 25,000 rpm for $2 \mathrm{~h}$. The supernatant was discarded, and the pellet containing the virus was resuspended with a virus preservation solution. The solution was then centrifuged at 10,000 rpm for $5 \mathrm{~min}$, and the supernatant was stored in a $-80^{\circ} \mathrm{C}$ ultralow freezer.

2.5. Lentivirus Infection. The A549 cells were seeded on a 24well plate at a density of $5-8 \times 10^{4}$ cells/well and infected with $1 \times 10^{8} \mathrm{TU} / \mathrm{mL}, 1 \times 10^{7} \mathrm{TU} / \mathrm{mL}$, and $1 \times 10^{6} \mathrm{TU} / \mathrm{mL}$ for $48 \mathrm{~h}$. A control cell line (NC-A549) without lentivirus infection was also maintained. The stable transfected cells were then screened using puromycin. Trypsin was used to dissociate cells into a single-cell suspension. The cells were then seeded onto a 96-well plate to obtain a cellular monolayer in order to establish cell lines.

2.6. Cell Viability Assay. A549 cells were seeded onto 96-well plates at a density of 7,000 cells/well and maintained for $48 \mathrm{~h}$. Following which, the CCK-8 solution (Dojindo, Japan) was carefully added to the cells $(100 \mu \mathrm{L}$ per well). The plates were then incubated in the dark for $2 \mathrm{~h}$ at $37^{\circ} \mathrm{C}$. The absorbance was periodically detected at $450 \mathrm{~nm}$ using a microplate reader.

2.7. Cell Apoptosis Assay. A549 cell apoptosis assay was performed via flow cytometry using an Annexin V/PI Apoptosis Kit (BD, Franklin Lakes, NJ, USA). A549 cells were collected following the transfection of miR-296-5p lentiviral vector or empty lentiviral vector for $48 \mathrm{~h}$; then, these cells were then labeled using Annexin V and PI according to the manufacturer's instruction. Flow cytometry assay was performed to detect the percentage of early apoptotic cells.

2.8. RT-qPCR Analysis for RNA Expression. Total RNA was extracted using TRIzol (Invitrogen, Carlsbad, CA, USA) according to the manufacturer's instruction. RT-qPCR was performed using Fast Start Universal SYBR Green Master (Roche). U6 was used as an endogenous control. Fold changes in the mRNA expression levels normalized to GAPDH were calculated using the $2^{-\Delta \Delta \mathrm{ct}}$ method. The following primers were used: U6 forward, $5^{\prime}$-CTCGCTTCG
GCAGCACA-3'; U6 reverse, 5' -AACGCTTCACGAATTT GCGT-3; GAPDH forward, 5'-CCTCAAGATCATCAGC AATGCCTC-3'; GAPDH reverse, $5^{\prime}$-GTGGTCATGAG TCCTTCCACGATA-3'; miR-296-5p forward, $5^{\prime}$-TGCC TAATTCAGAGGGTTGG-3' ${ }^{\prime}$; and miR-296-5p reverse, $5^{\prime}$ -CTCCACTCCTGGCACACAG-3'.

2.9. Protein Extraction and Western Blotting. The A549 cells were cultured for $48 \mathrm{~h}$. The cells were then washed twice with PBS. Radioimmunoprecipitation assay buffer (RIPA; Beyotime, Shanghai, China) containing $1 \mathrm{mM}$ phenylmethanesulfonylfluoride (PMSF; Beyotime, Shanghai, China) was added, and the cells were detached using a cell scraper. The solution was then centrifuged for $20 \mathrm{~min}$ at $12,000 \mathrm{rpm}$ at $4^{\circ} \mathrm{C}$ and further lysed using ultrasound. Protein concentrations were quantified using the BCA protein assay kit (Beyotime, Shanghai, China). Cell lysates were boiled for $10 \mathrm{~min}$ at $95^{\circ} \mathrm{C}$ and stored at $-80^{\circ} \mathrm{C}$. Total proteins $(20 \mu \mathrm{g})$ were electrophoresed on SDS-PAGE gels using the Miniprotein III system (BioRad, California, USA) and were then transferred to PVDF membranes (Millipore, USA) for $2 \mathrm{~h}$, followed by overnight incubation with primary antibody against WNT7B, Catenin-beta, SFTPA1 (SP-A), SP-B, or GAPDH $(1: 1000$; Affinity Biosciences, Cincinnati, OH, USA) at $4^{\circ} \mathrm{C}$. The following day, PVDF membranes were washed three times using PBST solution and were incubated at room temperature for $1 \mathrm{~h}$ in peroxidase-conjugated goat antirabbit IgG secondary antibodies (1:5,000; Bioworld Technology, Co. Ltd., USA). Further, the membrane was washed three times and processed for chemiluminescence using the Immobilon Western Chemiluminescent HRP Substrate Kit (Millipore, USA).

2.10. Statistical Analysis. Numerical data were reported as mean \pm SD. The GraphPad Prism version 5 software program was used for statistical analyses. Continuous variables were analyzed using Student's $t$-test or Mann-Whitney $U$ test to compare differences among groups. $p<0.05$ was considered significant.

\section{Results}

3.1. miR-296-5p Was Overexpressed in the Serum of Premature Infants with NRDS. To determine the expression levels of miR-296-5p in preterm infants with NRDS, we collected the serum samples from 13 preterm infants without NRDS and 10 preterm infants with NRDS. As shown in Figure 1, the values of miR-296-5p were $1.04 \pm 0.69$ and $3.16 \pm 3.35$, respectively, and the difference between the two groups was statistically significant $(p=0.04)$, indicating the overexpression of mir-296-5p in NRDS preterm infants as compared to non-NRDS preterm infants.

3.2. Establish Overexpression of $m i R-296-5 p$ in A549 Cell Lines. To elucidate the role of miR-296-5p in NRDS, we constructed lentiviral miR-296-5p overexpression cell lines (hereafter "miR-296-5p group"). Following the transfection of the miR-296-5p lentiviral vector into A549 cells, fluorescence microscopy demonstrated that the transfection rate 


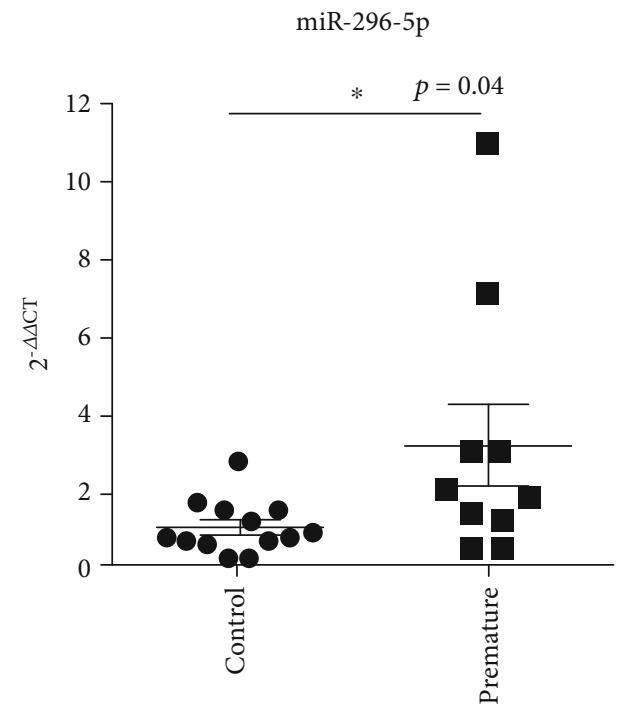

FIGURE 1: miR-296-5p was highly expressed in the sera of premature infants with NRDS. The sera from 13 premature infants without NRDS and 10 premature infants with NRDS were collected, and then, RT-qPCR assay was used to detect miR-296-5p expression. * $p<0.05$.

was greater than $85 \%$, as shown in Figure 2(a). The expression levels of miR-296-5p in NC-A549 and A549 cells were detected using RT-qPCR. The expression of miR-296-5p in the miR-296-5p group was significantly higher than that in the NC-A549 group $(p=0.011)$ (Figure 2(b)).

3.3. miR-296-5p Inhibited the Proliferation of A549 Cells with Unchanged Apoptosis. To study the effect of miR-296-5p on the A549 cells, we cultured the overexpressed miR-296-5p, NC-A549, and A549 cells for 24, 48, and $72 \mathrm{~h}$. Further, apoptosis and proliferation of A549 cells were examined using flow cytometry and CCK8 assay, respectively. The results of the Annexin-V/PI apoptosis assay showed that the percentage of apoptotic cells (Annexin-V+PI-) was $0.9 \%$ in the miR-296-5p group and $1 \%$ in the NC group $(p>0.05)$ (Figures 3(a) and 3(b)). The data of the CCK8 assay showed that cell viability was significantly lower in the miR-296-5p group compared to the NC group $(p<0.001)$ (Figure $3(c)$ ). These results suggest that miR-296-5p may inhibit the proliferation of A549 cells. However, miR-296-5p inhibition did not induce apoptosis in vitro.

3.4. miR-296-5p May Inhibit the Expression of SP-A and SP-B through the WNT Signaling Pathway. PS plays a crucial role in the maintenance of normal lung function in neonates. Lack of PS has been implicated in NRDS. Surfactant protein A (SP-A, SFTPA1) and surfactant protein B (SP-B) are the most important components of PS. To explore the potential mechanism of miR-296-5p in A549 cells, we determined the ratio of SP-A to SP-B among the NC and miR-296-5P groups via western blotting and found them to be 1.67 and 2.25, respectively (Figure 4). These results indicate that miR-296-5p inhibits the production of SP-A and SP-B in A549 cells. Wnt7b is one of the target genes of miR-296-5p.
Therefore, in this study, we investigated the role of the Wnt7b/ $\beta$-catenin pathway in NRDS using western blot. The ratios of $\mathrm{Wnt} 7 \mathrm{~b}$ and $\beta$-catenin among the $\mathrm{NC}$ and miR-296-5p groups were 2.22 and 1.45 , respectively (Figure 4). These results suggest that miR-296-5p may inhibit SP-A and SP-B through the Wnt7b pathway in A549 cells.

\section{Discussion}

PS is primarily composed of phospholipids that could reduce alveolar surface tension and maintain the effective exchange of blood gases. Surfactant proteins SP-A, SP-B, SP-C, and SP-D are known to be crucial components of alveolar surfactant. Each of them makes contributions to lung homeostasis through their primary protein structures and activities [38]. Mutations in the genes encoding SP-B (SFTPB) and SP-C (SFTPC) have been identified as pathogenic factors in fullterm infants with refractory respiratory failure after birth $[39,40]$. NRDS is pathologically characterized by progressive dyspnea and respiratory failure due to inadequate secretion of surfactant lipids and proteins by immature AECII. Expanding the knowledge of surfactant proteins and lipids can elucidate improved applications to prevent and treat NRDS.

Recent studies have shown that miRNAs are involved in the developmental process of lung maturation. Bhaskaran et al. identified significant changes in expression of $21 \mathrm{miR}$ NAs during lung development with a miRNA microarray and demonstrated that miR-127 had the greatest expression during the late stage of fetal lung development [7]. The expression of miR-431 was demonstrated to decline in preterm infants without RDS compared with those with RDS [41]. In vitro cell experiments show that miR-431 restricts the expression of SP proteins through suppression of the $\mathrm{BMP} 4 /$ activin/transforming growth factor- $\beta$ signaling pathway by targeting SMAD4 in A549 cells [42]. In the present study, the expression of miR-296-5p in preterm infants with NRDS was greater than in premature infants without NRDS. The sample size was small however; thus, we need to expand the sample size in future experiments. Würdinger et al. [21] demonstrated that miR-296-5p promotes the expression of growth factor receptors in vascular epithelial cells and affects tyrosine kinase substrates via regulation of hepatocyte growth factor. This results in a reduced number of blood vessels and induction of degradation of receptors for endothelial growth factor and platelet-derived growth factor. We found the ratio of SP-A to SP-B in the NC and miR-296-5P groups to be 1.67 and 2.25 , respectively. This suggests that miR-296$5 \mathrm{p}$ may inhibit the expression of SP proteins in A549 cells.

$\mathrm{Wnt} / \beta$-catenin signaling plays an important role in the differentiation and development of fetal lungs [43, 44]. Clevers et al. [45] demonstrated that Wnt signaling in the mouse- and human-induced pluripotent stem cells is an effective regulator of proximal and distal epithelial patterns. Wnt7b is mainly located in proximal and distal bronchial epithelial cells [46], and Wnt7b downstream $\beta$-catenin is restricted in alveolar and airway epithelial cells [47]. Murine model studies have shown that $\mathrm{Wnt} / \beta$-catenin signaling is imperative for the proliferation of airway submucosal gland 


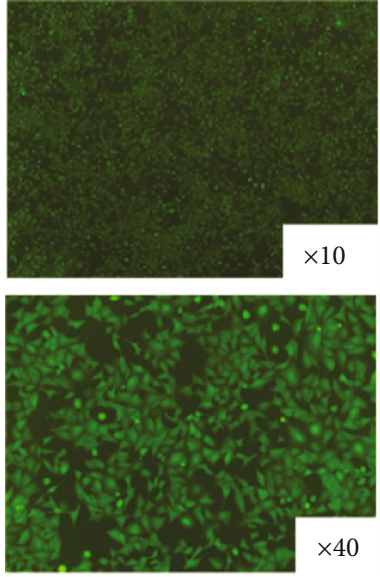

NC
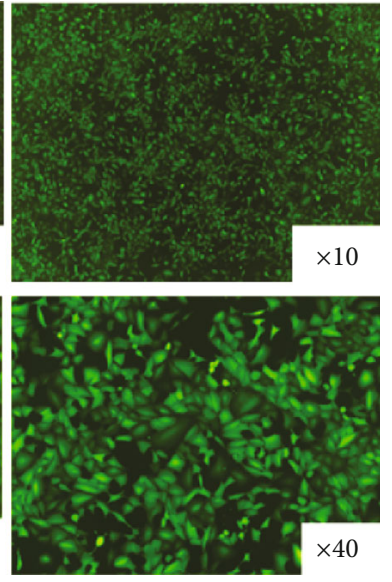

miR-296-5p

(a)

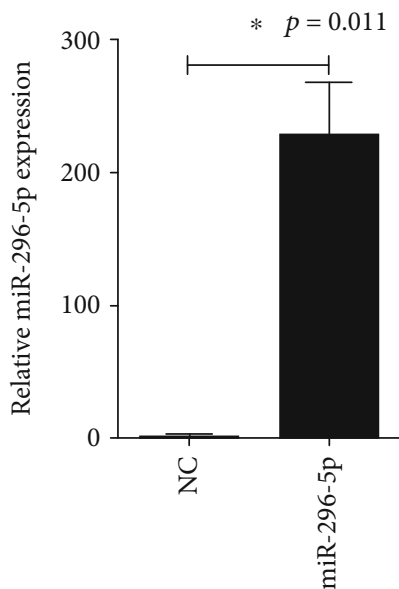

(b)

FIGURE 2: Lentiviral vector-mediated transfection was used to establish stable overexpression of miR-296-5p in A549 cell lines. (a). The transfection efficiency of lentivirus mir-296-5p in A549 cells was detected using fluorescence. (b). The RT-qPCR assay was used to detect miR-296-5p expression after transfection. ${ }^{*} p<0.05$.
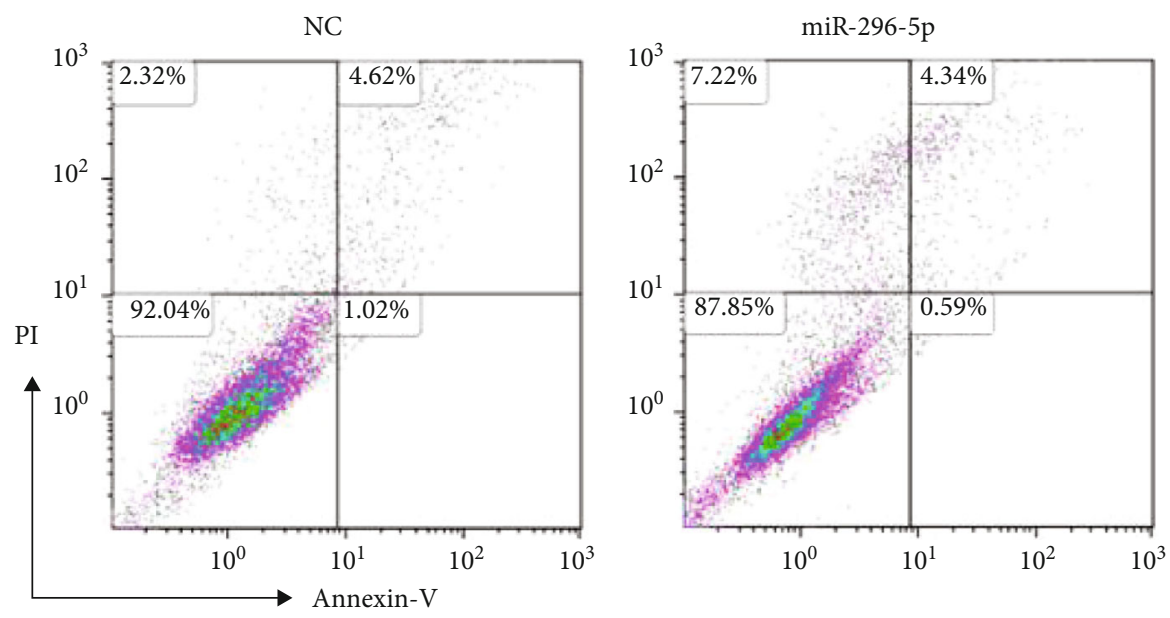

(a)

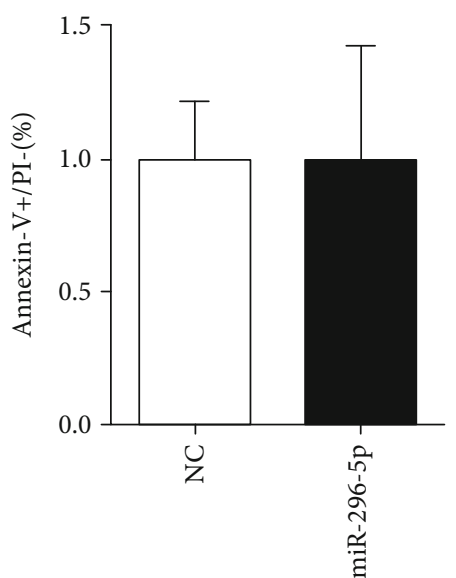

(b)

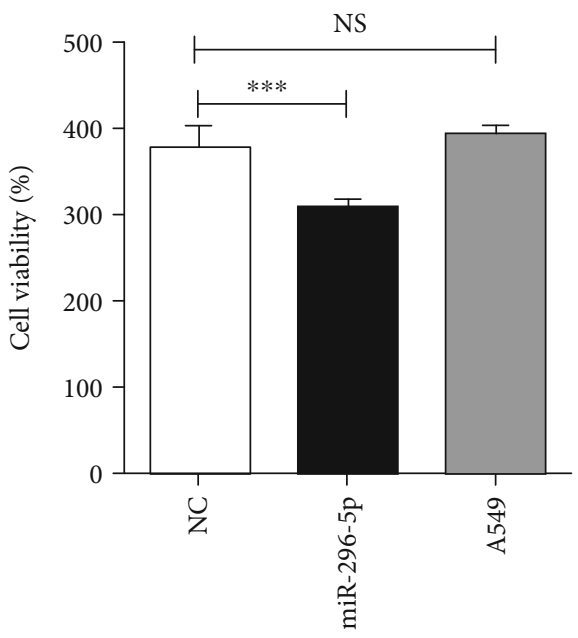

(c)

FIGURE 3: miR-296-5p inhibited proliferation of A549 cells with unchanged apoptosis. (a), (b). The apoptosis of A549 cells was analyzed using the apoptosis assay. (c). The cell viability of A549 cells was detected using the CCK-8 assay. ${ }^{* * *} p<0.001$. 


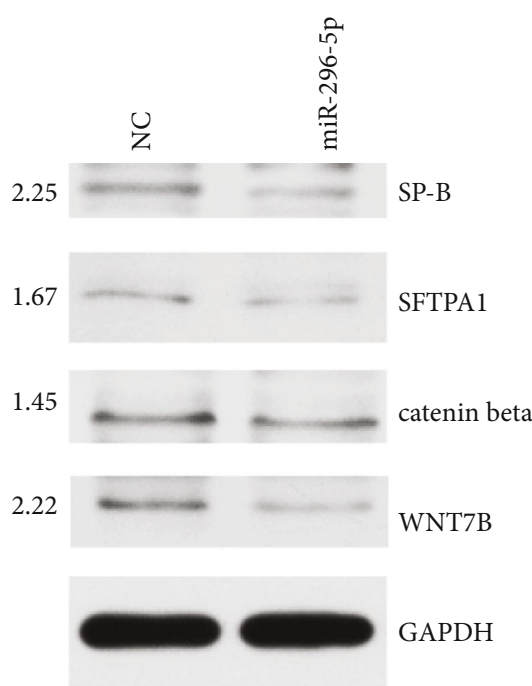

FIGURE 4: miR-296-5p inhibited the expression of SP-A (SFTPA1) and SP-B through the Wnt signaling pathway. After transfection of mir-296-5p overexpression and empty vectors in the A549 cell line, the expression of WNT7B, $\beta$-catenin, SP-A, and SP-B was assessed using western blotting.

progenitor cells, and the deletion and/or worsening of $\mathrm{Wnt} / \beta$-catenin signaling in the early development of lung epithelium cells leads to severe disruption of mesenchymal and epithelial compartments. This subsequently leads to the attenuation of secondary and tertiary branching [47] and hyperplasia and hypertrophy of submucosal glands [48]. However, in the present study, the ratios of Wnt7b and $\beta$ catenin in the NC and miR-296-5p groups were 2.22 and 1.45 , respectively. This suggests that miR-296-5p may inhibit Wnt/ $\beta$-catenin signaling in A549 cells.

There are some limitations to our study. AECII have certain stem cell characteristics including the ability to automatically transdifferentiate during in vitro culture. The intervention conditions of AECII are complex, which does not provide sufficient time to carry out the study accordingly. Therefore, we introduced a cell line, A549, as a replacement for AECII. The A549 cell line is a classic human AECII, containing lamellar bodies to produce surfactant and has a phospholipid content similar to that of AECII in situ [49]. We only detected proliferation and pulmonary surfactant secretion by A549 cells after transfection of the cells with miR296-5p. According to our studies to date, we suggest that miR-296-5p could be involved in the occurrence and development of NRDS via Wnt/ $\beta$-catenin signaling. However, we did not assess the status of other miR-296-5p target genes as well. We also did not further verify the biological function of miR-296-5p in vivo. These limited findings require further studies to clarify the underlying molecular mechanism.

\section{Conclusion}

In summary, the present study revealed that miR-296-5p levels were elevated in NRDS, which suppressed the proliferation and secretion of pulmonary surfactants in A549 cells by downregulating $\mathrm{Wnt7b} / \beta$-catenin signaling. Hence, the results of the present study hint at a novel molecular mechanism in which miR-296-5p is overexpressed in NRDS thereby deactivating $\mathrm{Wnt} 7 \mathrm{~b} / \beta$-catenin signaling.

\section{Data Availability}

The datasets used and/or analyzed during the current study are available from the corresponding author on reasonable request.

\section{Conflicts of Interest}

The authors have declared that no conflict of interest exists.

\section{Authors' Contributions}

Ying-Hui Zhang, Ai-Ling Chen, and Ren-Qiang Yu contributed equally to the work.

\section{Acknowledgments}

This work was supported by the funding from the scientific research project of Wuxi Commission of Health and Family Planning (grant number Q201742), Wuxi Young and Middle-aged Medical Talents Project (BJ2020075 and BJ2020079), and Wuxi Key Medical Discipline (ZDXK003).

\section{References}

[1] D. G. Sweet, V. Carnielli, G. Greisen et al., "European consensus guidelines on the management of respiratory distress syndrome-2016 update," Neonatology, vol. 111, no. 2, pp. 107-125, 2016.

[2] M. M. W. Chong, G. Zhang, S. Cheloufi, T. A. Neubert, G. J. Hannon, and D. R. Littman, "Canonical and alternate functions of the microRNA biogenesis machinery," Genes \& development, vol. 24, no. 17, pp. 1951-1960, 2010.

[3] M. Esteller, "Non-coding RNAs in human disease," Nature Reviews Genetics, vol. 12, no. 12, pp. 861-874, 2011.

[4] C. P. Bracken, H. S. Scott, and G. J. Goodall, "A networkbiology perspective of microRNA function and dysfunction in cancer," Nature Reviews Genetics, vol. 17, pp. 719-732, 2016.

[5] D. Ameis, N. Khoshgoo, B. M. Iwasiow, P. Snarr, and R. Keijzer, "MicroRNAs in lung development and disease," Paediatric Respiratory Reviews, vol. 22, pp. 38-43, 2017.

[6] A. E. Williams, S. A. Moschos, M. M. Perry, P. J. Barnes, and M. A. Lindsay, "Maternally imprinted microRNAs are differentially expressed during mouse and human lung development," Developmental Dynamics, vol. 236, no. 2, pp. 572580, 2007.

[7] M. Bhaskaran, Y. Wang, H. Zhang et al., "MicroRNA-127 modulates fetal lung development," Physiological Genomics, vol. 37, no. 3, pp. 268-278, 2009.

[8] J. Dong, G. Jiang, Y. W. Asmann et al., "MicroRNA networks in mouse lung organogenesis," PLoS One, vol. 5, no. 5, article e10854, 2010.

[9] K. S. Harris, Z. Zhang, M. T. McManus, B. D. Harfe, and X. Sun, "Dicerfunction is essential for lung epithelium morphogenesis," Proceedings. National Academy of Sciences. United States of America, vol. 103, no. 7, pp. 2208-2213, 2006. 
[10] Y. Lu, J. M. Thomson, H. Y. Wong, S. M. Hammond, and B. L. Hogan, "Transgenic over-expression of the microRNA_miR17-92_ cluster promotes proliferation and inhibits differentiation of lung epithelial progenitor cells," Developmental Biology, vol. 310, no. 2, pp. 442-453, 2007.

[11] A. Rodriguez, E. Vigorito, S. Clare et al., "Requirement of bic/microRNA-155 for normal immune function," Science, vol. 316, no. 5824, pp. 608-611, 2007.

[12] Y. H. Zhang, Y. Yang, C. Zhang et al., "Prediction of microRNA-296-5p target genes and its application in lung development," Zhongguo Dang Dai Er Ke Za Zhi, vol. 18, no. 12, pp. 1302-1307, 2016.

[13] K.-H. Lee, F.-C. Lin, T.-I. Hsu et al., "MicroRNA-296-5p (miR-296-5p) functions as a tumor suppressor in prostate cancer by directly targeting Pin1," Biochimica et Biophysica Acta, vol. 1843, no. 9, pp. 2055-2066, 2014.

[14] J. J. Wei, X. Wu, Y. Peng et al., "Regulation of HMGA1 expression by microRNA-296 affects prostate cancer growth and invasion," Clinical Cancer Research, vol. 17, no. 6, pp. 12971305, 2011.

[15] C. Xu, S. Li, T. Chen et al., "miR-296-5p suppresses cell viability by directly targeting PLK1 in non-small cell lung cancer," Oncology Reports, vol. 35, no. 1, pp. 497-503, 2016.

[16] F. Savi, I. Forno, A. Faversani et al., "miR-296/Scribble axis is deregulated in human breast cancer and miR-296 restoration reduces tumour growth in vivo," Clinical Science, vol. 127, no. 4, pp. 233-242, 2014.

[17] V. Vaira, A. Faversani, T. Dohi et al., "miR-296 regulation of a cell polarity-cell plasticity module controls tumor progression," Oncogene, vol. 31, no. 1, pp. 27-38, 2012.

[18] T. Li, Y. Y. Lu, X. D. Zhao et al., "MicroRNA-296-5p increases proliferation in gastric cancer through repression of Caudalrelated homeobox 1," Oncogene, vol. 33, no. 6, pp. 783-793, 2014.

[19] L. Hong, Y. Han, S. Li et al., "Role of ZNRD1 (zinc ribbon domain-containing 1) in angiogenesis of leukaemia cells," Cell Biology International, vol. 35, no. 4, pp. 321-324, 2011.

[20] X. Q. Liu, W. J. Song, T. M. Sun, P. Z. Zhang, and J. Wang, "Targeted delivery of antisense inhibitor of miRNA for antiangiogenesis therapy using cRGD-functionalized nanoparticles," Molecular Pharmaceutics, vol. 8, no. 1, pp. 250-259, 2011.

[21] T. Würdinger, B. A. Tannous, O. Saydam et al., "miR-296 regulates growth factor receptor overexpression in angiogenic endothelial cells," Cancer Cell, vol. 14, no. 5, pp. 382-393, 2008.

[22] H. Lee, S. J. Hwang, H. R. Kim et al., "Neurofibromatosis 2 (NF2) controls the invasiveness of glioblastoma through YAP-dependent expression of CYR61/CCN1 and miR-2963p," Biochimica et Biophysica Acta, vol. 1859, no. 4, pp. 599611, 2016.

[23] H. Lee, C. H. Shin, H. R. Kim, K. H. Choi, and H. H. Kim, "MicroRNA-296-5p promotes invasiveness through downregulation of nerve growth factor receptor and caspase-8," Molecules and Cells, vol. 40, no. 4, pp. 254-261, 2017.

[24] X. Deng, Z. Liu, X. Liu et al., "miR-296-3p negatively regulated by nicotine stimulates cytoplasmic translocation of c-Myc via MK2 to suppress chemotherapy resistance," Molecular Therapy, vol. 26, no. 4, pp. 1066-1081, 2018.

[25] N. Shivapurkar, S. Mikhail, R. Navarro et al., "Decrease in blood miR-296 predicts chemotherapy resistance and poor clinical outcome in patients receiving systemic chemotherapy for metastatic colon cancer," International Journal of Colorectal Disease, vol. 28, no. 6, 2013.

[26] W. Luo, Y. Lin, S. Meng, Y. Guo, J. Zhang, and W. Zhang, "miRNA-296-3p modulates chemosensitivity of lung cancer cells by targeting CX3CR1," American Journal of Translational Research, vol. 8, no. 4, pp. 1848-1856, 2016.

[27] Y. Tay, J. Zhang, A. M. Thomson, B. Lim, and I. Rigoutsos, "MicroRNAs to Nanog, Oct4 and Sox 2 coding regions modulate embryonic stem cell differentiation," Nature, vol. 455, no. 7216, pp. 1124-1128, 2008.

[28] L. Wang, W. Su, W. du et al., "Gene and microRNA profiling of human induced pluripotent stem cell-derived endothelial cells," Stem Cell Reviews, vol. 11, no. 2, article 9582, pp. 219227, 2015.

[29] A. E. Bishop, "Pulmonary epithelial stem cells," Cell Proliferation, vol. 37, no. 1, pp. 89-96, 2004.

[30] F. Fang, Z. Li, Q. Zhao, C. Xiong, and K. Ni, “Analysis of multilineage gene expression dynamics during primordial germ cell induction from human induced pluripotent stem cells," Stem Cell Research \& Therapy, vol. 11, no. 1, 2020.

[31] K. Kamizaki, M. Endo, Y. Minami, and Y. Kobayashi, "Role of noncanonical Wnt ligands and Ror-family receptor tyrosine kinases in the development, regeneration, and diseases of the musculoskeletal system," Developmental Dynamics, pp. 1-12, 2020.

[32] C. Wang, Y. Li, K. Yu, Z. Jiang, Y. Wang, and G. Yang, "HOXA10 inhibit the osteogenic differentiation of periodontal ligament stem cells by regulating $\beta$-catenin localization and DKK1 expression," Connective Tissue Research, pp. 1-9, 2020.

[33] H. Clevers and R. Nusse, "Wnt/ $\beta$-catenin signaling and disease," Cell, vol. 149, no. 6, pp. 1192-1205, 2012.

[34] M. Hussain, C. Xu, M. Lu, X. Wu, L. Tang, and X. Wu, "Wnt/ $\beta$-catenin signaling links embryonic lung development and asthmatic airway remodeling," Biochimica et Biophysica Acta, vol. 1863, no. 12, pp. 3226-3242, 2017.

[35] Q. Yu, L. Liu, Y. Duan et al., "Wnt/ $\beta$-catenin signaling regulates neuronal differentiation of mesenchymal stem cells," Biochemical and Biophysical Research Communications, vol. 439, no. 2, pp. 297-302, 2013.

[36] R. Olivares-Navarrete, S. L. Hyzy, J. H. Park et al., "Mediation of osteogenic differentiation of human mesenchymal stem cells on titanium surfaces by a Wnt-integrin feedback loop," Biomaterials, vol. 32, no. 27, pp. 6399-6411, 2011.

[37] T. Weng and L. Liu, "The role of pleiotrophin and $\beta$-catenin in fetal lung development," Respiratory Research, vol. 11, no. 1, 2010.

[38] J. A. Whitsett, S. E. Wert, and T. E. Weaver, "Alveolar surfactant homeostasis and the pathogenesis of pulmonary disease," Annual Review of Medicine, vol. 61, no. 1, pp. 105-119, 2010.

[39] S. E. Wert, J. A. Whitsett, and L. M. Nogee, "Genetic disorders of surfactant dysfunction," Pediatric and Developmental Pathology, vol. 12, no. 4, pp. 253-274, 2011.

[40] M. K. Dishop, "Paediatric interstitial lung disease: classification and definitions," Paediatric Respiratory Reviews, vol. 12, no. 4, pp. 230-237, 2011.

[41] K. Qing, Y. Yang, and X.-Y. Zhou, "Expression profile of microRNAs in fetal lung development of Sprague-Dawley rats," International Journal of Molecular Medicine, vol. 29, no. 3, pp. 393-402, 2012.

[42] Y. Q. Shen, Z. D. Bao, J. J. Pan et al., "MicroRNA-431 inhibits the expression of surfactant proteins through the 
BMP4/activin/TGF- $\beta$ signaling pathway by targeting SMAD4," International Journal of Molecular Medicine, 2020.

[43] J. H. Lee and E. L. Rawlins, "Developmental mechanisms and adult stem cells for therapeutic lung regeneration," Developmental Biology, vol. 433, no. 2, pp. 166-176, 2018.

[44] H. Clevers, K. M. Loh, and R. Nusse, "An integral program for tissue renewal and regeneration: Wnt signaling and stem cell control," Science, vol. 346, no. 6205, article 1248012, 2014.

[45] K. B. McCauley, F. Hawkins, M. Serra, D. C. Thomas, A. Jacob, and D. N. Kotton, "Efficient Derivation of functional human airway epithelium from pluripotent stem cells via temporal regulation of Wnt signaling," Cell Stem Cell, vol. 20, no. 6, pp. 844-857.e6, 2017.

[46] J. Weidenfeld, W. Shu, L. Zhang, S. E. Millar, and E. E. Morrisey, "The WNT7b promoter is regulated by TTF-1, GATA6, and Foxa2 in lung epithelium," The Journal of Biological Chemistry, vol. 277, no. 23, pp. 21061-21070, 2002.

[47] C. G. Eberhart and P. Argani, "Wnt signaling in human development: beta-catenin nuclear translocation in fetal lung, kidney, placenta, capillaries, adrenal, and cartilage," Pediatric and Developmental Pathology, vol. 4, no. 4, pp. 351-357, 2001.

[48] R. R. Driskell, M. Goodheart, T. Neff et al., "Wnt3a regulates Lef- 1 expression during airway submucosal gland morphogenesis," Developmental Biology, vol. 305, no. 1, pp. 90-102, 2007.

[49] M. B. Ryndak, K. K. Singh, Z. Peng, and S. Laal, "Transcriptional profile of mycobacterium tuberculosis replicating in type II alveolar epithelial cells," PLoS One, vol. 10, no. 4, article e0123745, 2015. 